\title{
ON THE CLASSIFICATION OF ISOTROPIC TENSORS
}

\author{
by P. G. APPLEBY, B. R. DUFFY and R. W. OGDEN
}

(Received 14 January, 1986)

A tensor is said to be isotropic relative to a group of transformations if its components are invariant under the associated group of coordinate transformations. In this paper we review the classification of tensors which are isotropic under the general linear group, the special linear (unimodular) group and the rotational group. These correspond respectively to isotropic absolute tensors $[4,8]$, isotropic relative tensors [4] and isotropic Cartesian tensors [3]. New proofs are given for the representation of isotropic tensors in terms of Kronecker deltas and alternating tensors. And, for isotropic Cartesian tensors, we provide a complete classification, clarifying results described in [3].

In the final section of the paper certain derivatives of isotropic tensor fields are examined.

1. Introduction and notation. Let $X$ be a vector space of finite dimension $N \geqslant 2$ and let $X^{*}$ be its dual. A tensor $\mathbf{T}$ of contravariant order $U$ and covariant order $V$ is an element of the vector space

$$
\otimes_{V}^{U} X=\underbrace{X \otimes X \otimes \ldots \otimes X}_{U \text { times }} \otimes \underbrace{X^{*} \otimes X^{*} \otimes \ldots \otimes X^{*}}_{V \text { times }}
$$

of dimension $N^{U+V}$. We denote by $T_{j_{1} j_{2} \ldots j_{v}}^{i_{1} i_{2} \ldots i_{\nu}}$ the components of $\mathbf{T}$ with respect to a general basis $\left\{\mathbf{e}_{i}\right\}$ for $X$ and dual basis $\left\{\mathbf{e}^{i}\right\}$ for $X^{*}$. All indices run over values $1,2, \ldots, N$ and in what follows the usual Einstein summation convention is adopted.

The general linear group of transformations of $X$ to itself is denoted by $G L(N)$; each transformation $\mathbf{a} \in G L(N)$ defines an associated linear transformation

$$
\otimes_{V}^{U} X \rightarrow \otimes_{V}^{U} X, \quad \mathbf{T} \mapsto \mathbf{a T},
$$

such that for all $\mathbf{x}_{1}, \mathbf{x}_{2}, \ldots, \mathbf{x}_{U} \in X$ and all $\mathbf{y}_{1}, \mathbf{y}_{2}, \ldots, \mathbf{y}_{V} \in X^{*}$

$$
\mathbf{a}\left(\mathbf{x}_{1} \otimes \ldots \otimes \mathbf{x}_{U} \otimes \mathbf{y}_{1} \otimes \ldots \otimes \mathbf{y}_{V}\right)=\mathbf{a x}_{1} \otimes \ldots \otimes \mathbf{a x}_{U} \otimes \mathbf{y}_{\mathbf{1}} \mathbf{b} \otimes \ldots \otimes \mathbf{y}_{V} \mathbf{b}
$$

where $\mathbf{b}$ is the inverse of $\mathbf{a}$.

We call aT the image of $\mathbf{T}$ under $\mathbf{a}$. The components of $\mathbf{T}$ and aT relative to the basis $\left\{\mathbf{e}_{i}\right\}$ are related by

$$
(\mathbf{a T})_{j_{1} j_{2} \ldots j_{V}}^{i_{1} \ldots i_{U}}=\prod_{r=1}^{U} a_{m_{r}}^{i_{r}} \prod_{s=1}^{V} b_{j_{s}}^{n_{s}} T_{n_{1} n_{2} \ldots n_{V}}^{m_{1} m_{2} \ldots m_{U}},
$$

where the components $a_{j}^{i}$ are defined by $\mathbf{a} \mathbf{e}_{i}=a_{i}^{j} \mathbf{e}_{j}$ and $b_{j}^{i}$ by $\mathbf{e}^{i} \mathbf{b}=b_{j}^{i} \mathbf{e}^{j}$.

A tensor $\mathbf{T}$ is said to be isotropic relative to a group $\mathscr{G}$ of non-singular linear

Glasgow Math. J. 29 (1987) 185-196. 
transformations $X \rightarrow X$ if $\mathbf{T}$ is invariant under all transformations $\mathbf{a} \in \mathscr{G}$, that is

$$
\mathbf{T}=\mathbf{a} \mathbf{T} \quad \forall \mathbf{a} \in \mathscr{G}
$$

Since the components of aT relative to the transformed basis $\left\{\tilde{\mathbf{e}}_{i}\right\}$ and its dual $\left\{\tilde{\mathbf{e}}^{i}\right\}$, defined by

$$
\begin{aligned}
\tilde{\mathbf{e}}_{i} & =\mathbf{a \mathbf { e } _ { i }}=a_{i}^{j} \mathbf{e}_{j}, \\
\tilde{\mathbf{e}}^{i} & =\mathbf{e}^{i} \mathbf{b}=b_{j}^{i} \mathbf{e}^{j},
\end{aligned}
$$

are the same as the components of $\mathbf{T}$ relative to the basis $\left\{\mathbf{e}_{i}\right\}$, the components of $\mathbf{T}$ are invariant under the basis transformation $\mathbf{e}_{i} \mapsto \mathbf{a e}_{i}$ for all $\mathbf{a} \in \mathscr{G}$.

2. General isotropic tensors. Tensors isotropic under the general linear group $G L(N)$ have components that are invariant under all basis transformations. The basic properties of these tensors have been determined by Thomas [8] and Knebelman [4]. Here we review their results and present a new proof of the representation of general isotropic tensors.

First we note that any non-trivial general isotropic tensor must have equal contravariant and covariant orders $[8,4]$. If $\mathbf{T}$ is a tensor of type $(U, V)$ (that is, of contravariant order $U$ and covariant order $V$ ) then by setting $\mathbf{a}=\lambda \mathbf{1}$, where $\lambda \in \mathbb{R}-\{0\}$ and 1 denotes the identity transformation, we obtain from (1.1)

$$
\mathbf{a T}=\lambda^{U-V} \mathbf{T}
$$

Hence, if $\mathbf{T}$ is isotropic, we must have

$$
\mathbf{T}=\lambda^{U-V} \mathbf{T}
$$

for all $\lambda \neq 0$; hence $U=V$.

If $\mathbf{T}$ is a general isotropic tensor of type $(U, U)$ then its components must satisfy

$$
\prod_{r=1}^{U} a_{m_{r}}^{i_{r}} T_{j_{1} \ldots j_{U}}^{m_{1} \ldots m_{U}}=\prod_{r=1}^{U} a_{j_{r}}^{n_{r}} T_{n_{1} \ldots n_{U}}^{i_{1} \ldots i_{U}}
$$

for all $\mathbf{a} \in G L(i)$. Differentiating (2.1) with respect to $a_{q}^{p}$ and then setting $a_{q}^{p}=\delta_{q}^{p}$, where $\delta_{q}^{p}$ is the Kronecker delta, we obtain the result

$$
\delta_{p}^{i_{1}} T_{j_{1} j_{2} \ldots j_{U}}^{q_{i_{2} \ldots i_{U}}}+\delta_{p}^{i_{2}} T_{j_{1} j_{2} \ldots j_{U}}^{i_{1} \ldots \ldots i_{U}}+\ldots+\delta_{p}^{i_{U}} T_{j_{1} j_{2} \ldots j_{U}}^{i_{1} i_{2} \ldots q}=\delta_{j_{1}}^{q_{1}} T_{p j_{2} \ldots j_{U}}^{i_{1} i_{2} \ldots i_{U}}+\delta_{j_{2}}^{q} T_{j_{1} \ldots j_{U}}^{i_{1} i_{2} \ldots i_{U}}+\ldots+\delta_{j_{U}}^{q} T_{j_{1} j_{2} \ldots p}^{i_{1} i_{2} \ldots i_{U}}
$$

given by Thomas [8]. Thomas outlined a method in which a system of linear equations obtained from (2.2) were solved to show, by induction, that $\mathbf{T}$ can be written as the sum of products of $U$ Kronecker deltas.

A much shorter proof of Thomas's results can be obtained using the following generalization of (2.2). We consider first the case $U=2$, for which equation (2.1) becomes

$$
a_{k}^{i} a_{l}^{i} T_{p q}^{k l}=a_{p}^{m} a_{q}^{n} T_{m n}^{i j}
$$


Differentiation of this with respect to $a_{s}^{r}$ and $a_{v}^{u}$ in turn leads to

$$
\delta_{r}^{i} \delta_{u}^{j} T_{p q}^{s v}+\delta_{u}^{i} \delta_{r}^{j} T_{p q}^{v s}=\delta_{p}^{s} \delta_{q}^{v} T_{r u}^{i j}+\delta_{p}^{v} \delta_{q}^{s} T_{u r \cdot}^{i j}
$$

Contraction of $u$ with $v$ in (2.3) yields equation (2.2) for $U=2$.

We now generalize this formula by differentiating (2.1) with respect to each of $a_{q_{1}}^{p_{1}}, a_{q_{2}}^{p_{2}}, \ldots, a_{q_{U}}^{p_{U}}$ in turn; after some rearrangement, we obtain

$$
\sum_{\sigma \in S_{U}} \Delta_{\sigma\left(p_{1} p_{2} \ldots p_{U}\right)}^{i_{i} i_{2} \ldots i_{U}} T_{j_{1} j_{2} \ldots j_{U}}^{\sigma\left(q_{1} q_{2} \ldots q_{U}\right)}=\sum_{\sigma \in S_{U}} \Delta_{y_{1} j_{2} \ldots j_{U}}^{\sigma\left(q_{1} q_{2} \ldots q_{U}\right)} T_{\sigma\left(p_{1} p_{2} \ldots p_{U}\right)}^{i_{1} i_{2} \ldots i_{U}},
$$

where

$$
\Delta_{j_{1} j_{2} \ldots j_{U}}^{i_{1} i_{2} \ldots i_{U}}=\delta_{j_{1}}^{i_{1}} \delta_{j_{2}}^{i_{2}} \ldots \delta_{j_{U}}^{i_{U}}
$$

and the summations are over the set $S_{U}$ of all $U$ ! permutations $\sigma$ of $U$ indices, $\sigma\left(p_{1} p_{2} \ldots p_{U}\right)$ representing a permutation of $\left(p_{1}, p_{2}, \ldots, p_{U}\right)$.

Further identities can be obtained from (2.4) by contraction of 1 or 2 or up to $(U-1)$ indices $p_{r}$ with $q_{r}$. The latter of these alternatives recovers (2.2).

We now use (2.4) to obtain explicit forms for general isotropic tensors.

(i) For the case $U=1$ each of (2.2) and (2.4) reduces to

$$
\delta_{p}^{i} T_{j}^{q}=\delta_{j}^{q} T_{p}^{i}
$$

Setting $i=p=1$ and relabelling the indices, we obtain the well-known result

$$
T_{j}^{i}=\lambda \delta_{j}^{i},
$$

where $\lambda$ is an arbitrary scalar such that $\lambda=T_{1}^{1}=T_{2}^{2}=\ldots=T_{N}^{N}=N^{-1} T_{k^{*}}^{k}$

(ii) For $U=2$, we have from equation (2.3)

$$
T_{k l}^{i j}=\lambda \delta_{k}^{i} \delta_{l}^{j}+\mu \delta_{l}^{i} \delta_{k}^{j}
$$

after setting $i=r=1$ and $j=u=2$ and relabelling, where $\lambda$ and $\mu$ are arbitrary scalars such that

$$
\begin{aligned}
& \lambda=T_{12}^{12}=\left(N T_{p q}^{p q}-T_{p q}^{q p}\right) /\left(N^{3}-N\right), \\
& \mu=T_{21}^{12}=\left(N T_{p q}^{q p}-T_{p q}^{p q}\right) /\left(N^{3}-N\right) .
\end{aligned}
$$

Equation (2.7) gives the most general form for the components of an isotropic tensor of type $(2,2)$.

(iii) For the general case with $U \leqslant N$ we set $i_{r}=p_{r}=r(r=1,2, \ldots, U)$ in (2.4) and relabel suffices to obtain the result

$$
T_{j_{1} j_{2} \ldots j_{U}}^{i_{1} i_{2} \ldots i_{\nu}}=\sum_{\sigma \in S_{U}} \lambda_{\sigma} \Delta_{j_{1} j_{2} \ldots j_{U}}^{g\left(i_{1} i_{2}, \ldots i v\right)}
$$

where the $\lambda_{\sigma}$ are $U$ ! separate scalars given by

$$
\lambda_{\sigma}=T_{\sigma(12 \ldots U)}^{12 \ldots U} .
$$


(iv) The result (2.8) also holds when $U>N$, although the scalars $\lambda_{a}$ are not then given by (2.9). It suffices to consider the case $U=N+1$.

If $U=N+1$ then amongst the indices $q_{1}, q_{2}, \ldots, q_{N+1}$ at least one value in the set $\{1,2, \ldots, N\}$ must be repeated. Without loss of generality suppose $q_{N}=q_{N+1}$. Then, on selecting $i_{r}=p_{r}=r(1 \leqslant r \leqslant N), i_{N+1}=p_{N+1}=N$ we obtain from $(2.4)$

$$
T_{j_{1} \ldots j_{N+1}}^{q_{1} \ldots q_{N+1} q_{N+1}}+T_{j_{1} \ldots j_{N+1}}^{q_{1} \ldots q_{N+1} q_{N}}=\sum_{\sigma \in S_{U}} \Delta_{j_{1} \ldots j_{N+1}}^{\sigma\left(q_{1} \ldots q_{N+1}\right)} T_{\sigma(12 \ldots N N)}^{12 \ldots N}
$$

Since $q_{N}=q_{N+1}$ it follows that (2.8) holds with

$$
\lambda_{\sigma}=\frac{1}{2} T_{\sigma(12 \ldots N N)}^{12 \ldots N} \text {. }
$$

In a similar way (2.8) follows for $U \geqslant N+2$.

Note, however, that not all the components $\Delta_{j_{1} j_{2} \ldots j_{U}}^{i_{1} i_{2} \ldots i_{U}}$ are independent when $U>N$, since

$$
\sum_{\sigma \in S_{r}}(\operatorname{sgn} \sigma) \Delta_{j_{1} j_{2} \ldots j_{r}}^{\sigma\left(i_{1} i_{2} \ldots i_{r}\right)}=0 \quad(r \geqslant N+1) .
$$

This can be seen by noting that at least one index in the set $\{1,2, \ldots, N\}$ must be repeated in $i_{1}, i_{2}, \ldots, i_{r}$; interchange of an index with its repetition changes the sign of $\sigma$ but does not affect the value of $\Delta_{j_{1} j_{2} \ldots j_{r}}^{\sigma\left(i_{1} i_{2} \ldots i_{r}\right)}$. Alternatively, the result (2.10) can be obtained by making appropriate choices of $T_{j_{1} / 2 \ldots j_{U}}^{i_{1} i_{2} \ldots i_{U}}$ as products of Kronecker deltas in (2.4) and contracting.

It is worth noting that it follows from (2.10) that

$$
\sum_{\sigma \in S_{r}}(\operatorname{sgn} \sigma) T_{j_{1} \ldots j_{r}}^{\sigma\left(i_{1} \ldots i_{r}\right)}=0 \quad(r \geqslant N+1)
$$

for the components of any tensor $\mathbf{T}$ of type $(r, r)$.

3. Isotropy under the unimodular group. The unimodular (or special linear) group $S L(N)$ is the subgroup of transformations a $\in G L(N)$ such that

$$
\operatorname{det} \mathbf{a}=1 \text {. }
$$

A tensor $\mathbf{T}$ of type $(U, V)$ that is isotropic under this group must satisfy the invariance requirement (1.2) for all $\mathbf{a} \in S L(N)$.

Let $G L(N)^{+}$be the subgroup of $G L(N)$ consisting of transformations with positive determinant. Then, for each $\mathbf{a} \in G L(N)^{+}$there is an associated unimodular transformation ã defined by

$$
\tilde{\mathbf{a}}=(\operatorname{det} \mathbf{a})^{-1 / N} \mathbf{a} .
$$

From (1.1) we deduce that

$$
\tilde{\mathbf{a}} \mathbf{T}=(\operatorname{det} \mathbf{a})^{-[(U-V) / N]} \mathbf{a} \mathbf{T}
$$

and hence

$$
\tilde{\mathbf{a} T}=\mathbf{T} \text { for all } \tilde{\mathbf{a}} \in S L(N)
$$


if and only if

$$
(\operatorname{det} \mathbf{a})^{-W} \mathbf{a T}=\mathbf{T} \text { for all } \mathbf{a} \in G L(N)^{+},
$$

where

$$
W=(U-V) / N
$$

In index form (3.3) can be rearranged as

$$
T_{j_{1} \ldots j_{V}}^{i_{1} \ldots i_{U}}=(\operatorname{det} \mathbf{a})^{W} \prod_{r=1}^{V} a_{j_{r}}^{m_{r}} \prod_{s=1}^{U} b_{n_{s}}^{i_{s}} T_{m_{1} \ldots m_{V}}^{n_{1} \ldots n_{U}}
$$

The set of tensors $\mathbf{T}$ that are isotropic with respect to $S L(N)$ therefore characterizes the set of relative tensors $\dagger$ that are isotropic with respect to $G L(N)^{+}$.

We shall say that a tensor of type $(U, V)$ that is isotropic with respect to $S L(N)$ has weight $W$, given by (3.4).

It was shown in [4] that for a relative tensor to be isotropic (with respect to $\left.G L(N)^{+}\right) W$ must be an integer. We now provide a different proof of this result which yields some additional information. The case $N=3$ has been discussed in [5].

Let

$$
a_{j}^{i}=\lambda_{i} \delta_{j}^{i} \quad \text { (no summation), }
$$

where $0<\lambda_{i}<\infty$ for each $i \in\{1,2, \ldots, N\}$. Equation (3.5) then reduces to

$$
T_{j_{1} \ldots j_{V}}^{i_{1} \ldots i_{\nu}}=\prod_{r=1}^{N} \lambda_{r}^{W-U_{r}+V_{r}} T_{j_{1} \ldots j_{V}}^{i_{1} \ldots i_{\nu}}
$$

where $U_{r}$ (respectively $V_{r}$ ) is the number of times the index $r$ appears in the set $\left\{i_{1}, i_{2}, \ldots, i_{U}\right\}$ (respectively $\left\{j_{1}, j_{2}, \ldots, j_{V}\right\}$ ), so that $U=U_{1}+U_{2}+\ldots+U_{N}, V=V_{1}+$ $V_{2}+\ldots+V_{N}$. Since the $\lambda_{r}$ can be chosen independently, we deduce that

$$
W=U_{r}-V_{r} \quad \text { for each } r \in\{1,2, \ldots, N\},
$$

and hence $W$ is an integer (positive, negative or zero).

Equation (3.4) is recovered on summing equations (3.6) for $r=1$ to $N$. Equations (3.6) are apparently new. We note, in particular, that the only non-zero components of $\mathbf{T}$ are those for which $U_{r}-V_{r}=W$ for $r=1,2, \ldots, N$. For example, if $\mathbf{T}$ has equal covariant and contravariant order, so that $W=0$, then $U_{r}=V_{r}(r=1,2, \ldots, N)$; thus on a non-zero component $T_{j_{1} \ldots j u}^{i_{1} \ldots i \nu}$ the covariant indices are a permutation of the contravariant ones, a result that is also evident from (2.8). In this case $\mathbf{T}$ is isotropic with respect to $G L(N)^{+}$. If $\mathbf{T}$ is purely contravariant $(V=0)$ or covariant $(U=0)$ the order of $\mathbf{T}$ is $N|W|$ and on a non-zero component of $\mathbf{T}$ the indices must include each of the integers $1,2, \ldots, N$ exactly $|W|$ times; the particular case $|W|=1$ has special significance, as we see in what follows.

$\dagger$ The terminology density tensor is also used. See, for example, [2] and [7]. A relative tensor of weight $W$ with components $T_{j \ldots j}^{i \ldots j}$ with respect to the basis $\left\{\mathbf{e}_{i}\right\}$ has components with respect to $\left\{\overline{\boldsymbol{e}}_{i}\right\}$ given by the right-hand side of (3.5). We shall say that such a relative tensor is of type $(U, V, W)$. 
In considering isotropy under the unimodular group we may suppose, without loss of generality, that $X$ is an oriented vector space, the orientation being characterized by a particular $N$-covector (or volume element) $\mathbf{e} \in \wedge^{N} X^{*}$ (the space of $N$-covectors on $X$ ). This structure determines (see, for example, [1]) a special class of bases with respect to each of which the components of $\mathbf{e}$ are given by

$$
e_{i_{1} i_{2} \ldots i_{N}}=\left\{\begin{array}{l}
+1 \text { if } i_{1} i_{2} \ldots i_{N} \text { is an even permutation of }(1,2, \ldots, N) \\
-1 \text { if } i_{1} i_{2} \ldots i_{N} \text { is an odd permutation of }(1,2, \ldots, N) \\
0 \text { otherwise. }
\end{array}\right.
$$

The dual of $\mathbf{e}$ is an $N$-vector $\mathbf{e}^{*} \in \bigwedge^{N} X$ with components, denoted by $e^{i_{1} i_{2} \ldots i_{N}}$, having the same numerical values as $e_{i_{1} i_{2} \ldots i_{N}}$.

Since, for each $\mathbf{a} \in G L(N)$,

$$
\begin{aligned}
\mathbf{a e} & =(\operatorname{det} \mathbf{a})^{-1} \mathbf{e}, \\
\mathbf{a} \mathbf{e}^{*} & =(\operatorname{det} \mathbf{a}) \mathbf{e}^{*},
\end{aligned}
$$

it follows that $\mathbf{e}$ and $\mathbf{e}^{*}$ are isotropic tensors of order $N$ with respect to $S L(N)$, of weights -1 and +1 respectively.

From (3.3) or (3.5) we deduce that the tensor product of two tensors that are isotropic with respect to $S L(N)$ and of weights $W$ and $W^{\prime}$ is isotropic of weight $W+W^{\prime}$, and any contraction of a pair of co- and contravariant indices of an isotropic tensor yields an isotropic tensor of the same weight.

We now prove the following:

THEOREM (cf. Knebelman [4]). Any tensor T that is isotropic with respect to $S L(N)$ can be represented as the product of $|W|$ of the tensors $\mathbf{e}$ (or $\left.\mathbf{e}^{*}\right)$ and the general isotropic tensor with components given by (2.8).

(i) If $U>V$, and hence $W>0$, the tensor

$$
\mathbf{T} \otimes \underbrace{\mathbf{e} \ldots \otimes \mathbf{e}}_{W \text { times }}
$$

is isotropic of weight zero and type $(U, U)$, and can therefore be represented in the form (2.8). On use of the result

$$
e^{i_{1} \ldots i_{N}} e_{i_{1} \ldots i_{N}}=N !,
$$

which follows from the definition (3.7), we conclude that

$$
T_{j_{1} \ldots j_{V}}^{i_{1} \ldots i_{U}}=\sum_{\sigma \in S_{U}} \lambda_{\sigma} \Delta_{j_{1} \ldots j_{U}}^{\sigma\left(i_{1} \ldots i_{U}\right)} e^{j_{V+1} \ldots j_{V+N}} \ldots e^{j_{U-N+1} \ldots j_{U}}
$$

a factor $(N !)^{-W}$ having been absorbed by $\lambda_{\sigma}$.

(ii) If $U<V$ similar arguments to those used in (i) lead to

$$
T_{j_{1} \ldots j_{V}}^{i_{1} \ldots i_{U}}=\sum_{\sigma \in S_{V}} \lambda_{\sigma} \Delta_{\sigma\left(j_{1} \ldots j_{V}\right)}^{i_{1} \ldots i_{V}} e_{i_{U+1} \ldots i_{U+N}} \ldots e_{i_{V-N+1} \ldots i_{V}}
$$


Since

$$
\Delta_{j_{1} \ldots j_{N}}^{\sigma\left(i_{1} \ldots i_{N}\right)} e^{j_{1} \ldots j_{N}}=(\operatorname{sgn} \sigma) e^{i_{1} \ldots i_{N}}
$$

we may put $U=N, V=0$ in (3.9) to recover the well established result that the most general contravariant tensor isotropic of weight +1 under $S L(N)$ is given by

$$
\mathbf{T}=\lambda \mathbf{e}^{*} \text {, }
$$

where $\lambda$ is a scalar. This implies that $\lambda \mathrm{e}^{*}$ is the most general relative tensor, of type $(N, 0,1)$, isotropic under $G L(N)$. The corresponding result for the covariant case is obtained similarly.

The identity

$$
e^{i_{1} \ldots i_{N}} e_{j_{1} \ldots j_{N}}=\sum_{\sigma \in S_{N}}(\operatorname{sgn} \sigma) \Delta_{j_{1} \ldots j_{N}}^{\sigma\left(i_{1} \ldots i_{N}\right)},
$$

which will be used later, may also be deduced from the above theorem, using (3.9) and (2.9).

Finally in this section we note that equation (2.4) can be generalized for the case of tensors isotropic under $S L(N)$ by differentiating (3.5) with respect to $a_{q_{1}}^{p_{1}}, a_{q_{2}}^{p_{2}}, \ldots$ etc. in turn and then setting $a_{j}^{i}=\delta_{j}^{i}$. In particular, the generalization of (2.2) is

$$
\begin{aligned}
& \delta_{p}^{i_{1}} T_{j_{1} \ldots j_{V}}^{q_{2} \ldots i_{V}}+\delta_{p}^{i_{2}} T_{j_{1} \ldots j_{V}}^{i_{1} q_{1} i_{V}}+\ldots+\delta_{p}^{i_{\nu}} T_{j_{1} \ldots j_{V}}^{i_{1} i_{2} \ldots q}-\delta_{j_{1}}^{q} T_{p j_{2} \ldots j_{V}}^{i_{1} \ldots i_{\nu}}-\delta_{j_{2}}^{q} T_{j_{1} \ldots \ldots j_{V}}^{i_{1} \ldots i_{V}} \\
& -\ldots-\delta_{j_{v}}^{q} T_{j_{1} j_{2} \ldots p}^{i_{1} \ldots i_{U}}-W \delta_{p}^{q} T_{j_{1} \ldots j_{V}}^{i_{1} \ldots i_{U}}=0 .
\end{aligned}
$$

4. Isotropic Cartesian tensors. When considering isotropy with respect to the group $O(N)^{+}$of proper orthogonal transformations (or rotations) we may suppose, without loss of generality, that $X$ is a Euclidean vector space, that is $X$ has a positive definite scalar product. In this case we may identify $X$ canonically with its dual space $X^{*}$, and no distinction need be made between covariant and contravariant tensors. A tensor of order $U$ is simply an element of the vector space

$$
\otimes^{u} X=\underbrace{X \otimes \ldots \otimes X}_{U \text { times }}
$$

Provided we restrict attention to orthonormal basis vectors it is also unnecessary to distinguish between covariant and contravariant components. With this restriction a tensor $\mathbf{T} \in \otimes^{U} X$ is commonly referred to as a Cartesian tensor of order $U$. The (Cartesian) components of $\mathbf{T}$ are denoted by $T_{i_{1} \ldots i_{v}}$.

The invariance requirement (1.2) for an isotropic Cartesian tensor of order $U$ can be written [3]

where

$$
T_{i_{1} \ldots i_{U}}=a_{i_{1} j_{1}} a_{i_{2} j_{2}} \ldots a_{i_{u j u}} T_{j_{1} \ldots j_{U}}
$$

$$
\begin{gathered}
a_{i k} a_{j k}=a_{k i} a_{k j}=\delta_{i j} \\
\operatorname{det} \mathbf{a}=1 .
\end{gathered}
$$


From (3.8) $)_{1}$ the constraint (4.3) can also be expressed as

$$
a_{i_{1} j_{1}} a_{i_{2} j_{2}} \ldots a_{i_{N} j_{N}} e_{j_{1} \ldots j_{N}}=e_{i_{1} \ldots i_{N}}
$$

where $e_{i_{1} \ldots i_{N}}$ are the Cartesian components of the volume element e given by (3.7).

Examples of isotropic Cartesian tensors are the metric (or unit) tensor 1, with Cartesian components $\delta_{i j}$, and the tensor e itself. We write

$$
\Delta_{i_{1} j_{1} i_{2} j_{2} \ldots i_{U j} j_{U}}=\delta_{i_{1} j_{1}} \delta_{i_{2} j_{2}} \ldots \delta_{i_{U} j_{U}} .
$$

We now provide a new derivation of the form of the components $T_{i_{1} i_{2} \ldots i_{U}}$ of an isotropic Cartesian tensor, considering separately the cases of even and odd $U$.

(a) $U$ is even. Because of the constraints on the components $a_{i j}$ we cannot differentiate (4.1) as it stands along the lines used in Section 2 to obtain (2.4). We first need to incorporate the constraints in a suitable way with Lagrange multipliers.

From (4.2) we form an isotropic tensor constraint of order $U$, namely

$$
a_{i_{1} j_{1}} a_{i_{2} j_{2}} \ldots a_{i_{U} j_{U}} \sum_{\sigma \in S_{U}} \lambda_{\sigma} \Delta_{\sigma\left(j_{1} j_{2} \ldots j_{U}\right)}=\sum_{\sigma \in S_{U}} \lambda_{\sigma} \Delta_{\sigma\left(i_{1} i_{2} \ldots i_{U}\right)}
$$

where the $\lambda_{\sigma}$ are arbitrary scalars. If $N$ is odd no product of a single alternating symbol with Kronecker deltas will yield an isotropic tensor of order $U$ so that the constraint (4.4) is not required. We examine this case first.

(i) $N$ is odd. Equations (4.1) and (4.6) are combined to give the identity

$$
T_{i_{1} i_{2} \ldots i_{U}}-\sum_{\sigma \in S_{U}} \lambda_{\sigma} \Delta_{\sigma\left(i_{1} i_{2} \ldots i_{U}\right)}=a_{i_{1} j_{1}} a_{i_{2} j_{2}} \ldots a_{i_{U j} j_{U}}\left\{T_{j_{1} j_{2} \ldots j_{U}}-\sum_{\sigma \in S_{U}} \lambda_{\sigma} \Delta_{\sigma\left(j_{1} j_{2} \ldots j u\right)}\right\} .
$$

With the $\lambda_{\sigma}$ 's regarded as Lagrange multipliers we can now differentiate this with respect to $a_{m_{1} n_{1}}, a_{m_{2} n_{2}}, \ldots, a_{m_{U} n_{U}}$ in turn to give

$$
\sum_{\rho \in S_{U}} \delta_{i_{1} m_{r_{1}}} \delta_{i_{2} m_{r_{2}}} \ldots \delta_{i_{U} m_{r_{U}}}\left\{T_{n_{r_{1}, n_{r_{2}} \ldots n_{r_{U}}}}-\sum_{\sigma \in S_{U}} \lambda_{\sigma} \Delta_{\sigma\left(n_{r_{1}} n_{r_{2}} \ldots n_{r_{U}}\right)}\right\}=0
$$

where the first summation is over all permutations $r_{1} r_{2} \ldots r_{U}=\rho(12 \ldots U)$.

If $U<N$ then we may set $i_{\alpha}=m_{\alpha}=\alpha(1 \leqslant \alpha \leqslant U)$. The only non-vanishing contribution to the first sum in (4.7) therefore arises when $r_{\alpha}=\alpha(1 \leqslant \alpha \leqslant U)$, and (4.7) then simplifies to

$$
T_{n_{1} n_{2} \ldots n_{U}}=\sum_{\sigma \in S_{U}} \lambda_{\sigma} \Delta_{\sigma\left(n_{1} n_{2} \ldots n_{U}\right)}
$$

This result also holds when $U>N$, as can be shown by applying a similar argument to that used in the case of tensors of type $(U, U)$ in Section 2.

(ii) $N$ is even. If $U<N$ the most general form of isotropic Cartesian tensor again has components given by (4.8).

If $U=N$ the representation (4.8) is generalized to

$$
T_{n_{1} n_{2} \ldots n_{N}}=\sum_{\sigma \in S_{N}} \lambda_{\sigma} \Delta_{\sigma\left(n_{1} n_{2} \ldots n_{N}\right)}+\mu e_{n_{1} n_{2} \ldots n_{N}}
$$


where $\mu$ is an additional Lagrange multiplier in respect of the constraint (4.4). When $N=2$, for example, (4.9) becomes simply $T_{i j}=\lambda \delta_{i j}+\mu e_{i j}$.

If $U>N$ then we have the further generalization

$$
T_{n_{1} n_{2} \ldots n_{U}}=\sum_{\sigma \in S_{U}} \lambda_{\sigma} \Delta_{\sigma\left(n_{1} n_{2} \ldots n_{U}\right)}+\sum_{\sigma \in S_{U}} \mu_{\sigma} e_{\sigma\left(n_{1}\right) \sigma\left(n_{2}\right) \ldots \sigma\left(n_{N}\right)} \Delta_{\sigma\left(n_{N+1}\right) \sigma\left(n_{N+2}\right) \ldots \sigma\left(n_{U}\right)},
$$

where $\sigma\left(n_{1}\right) \sigma\left(n_{2}\right) \ldots \sigma\left(n_{U}\right)=\sigma\left(n_{1} n_{2} \ldots n_{U}\right)$ and the $\mu_{\sigma}$ are Lagrange multipliers. Note that because of (3.11), specialized to the Cartesian case, at most one $\mathbf{e}$ term is required in each term in the second summation in (4.10).

(b) $U$ is odd.

(i) $N$ is even. In this case we may choose $a_{i j}=-\delta_{i j}$ and (4.1) then yields

$$
T_{i_{1} i_{2} \ldots i_{U}}=(-1)^{U} T_{i_{1} i_{2} \ldots i_{U}}=-T_{i_{1} i_{2} \ldots i_{U}},
$$

i.e. $\mathbf{T}$ is necessarily the zero tensor.

(ii) $N$ is odd. We form the product

$$
R_{i_{1} i_{2} \ldots i_{U} j_{1} j_{2} \ldots j_{N}} \equiv T_{i_{1} i_{2} \ldots i_{U}} e_{j_{1} j_{2} \ldots j_{N}}
$$

to yield the components of an isotropic Cartesian tensor of even order $U+N$. By (a)(i) these components can be written in terms of products of $\frac{1}{2}(U+N)$ Kronecker deltas. On multiplying (4.11) by $e_{j_{1} j_{2} \ldots j_{N}}$ we obtain

$$
T_{i_{1} i_{2} \ldots i_{U}}=(N !)^{-1} R_{i_{1} i_{2} \ldots i_{U j} j_{j} \ldots j_{N}} e_{j_{1} j_{2} \ldots j_{N}}
$$

If $U<N$ then $U+2 \leqslant N$ and so, in $R_{i_{1} i_{2} \ldots i_{u j} j_{2} \ldots j_{N}}$, at least one pair of $j_{1}, j_{2}, \ldots, j_{N}$ must occur on the same Kronecker delta in each set of products. Hence $\mathbf{T}$ must be the zero tensor, as in (b)(i).

If $U=N$ then the only non-zero terms in (4.12) occur when each Kronecker delta has the form $\delta_{i_{r} j_{s}}$. Hence

$$
T_{i_{1} i_{2} \ldots i_{N}}=\lambda e_{i_{1} i_{2} \ldots i_{N}},
$$

where $\lambda$ is an absolute scalar, thus specializing the corresponding result given in Section 3 .

If $U>N$ then (4.12) yields

$$
T_{i_{1} i_{2} \ldots i_{U}}=\sum_{\sigma \in S_{N}} \mu_{\sigma} e_{\sigma\left(i_{1}\right) \sigma\left(i_{2}\right) \ldots \sigma\left(i_{N}\right)} \Delta_{\sigma\left(i_{N+1}\right) \sigma\left(i_{N+2}\right) \ldots \sigma\left(i_{U}\right)}
$$

as in the second summation in (4.10).

In conclusion we see that the most general form for the components of an isotropic Cartesian tensor is given by (4.10) with some or all of the coefficients being zero depending on whether $U$ and $N$ are even or odd.

For another proof see [3] and the references given therein. It is not clear from [3] that all the separate cases discussed here have been covered previously.

It is interesting to compare the results for Cartesian tensors with those for general tensors given in Section 2. In particular, for $U=4$ and $N$ odd we have, from (4.8),

$$
T_{i j k l}=\sum_{\sigma \in S_{4}} \lambda_{\sigma} \Delta_{\sigma(i j k l)}=\sum_{\sigma \in S_{4}} \lambda_{\sigma} \delta_{\sigma(i) \sigma(j)} \delta_{\sigma(k) \sigma(l)} .
$$


The 24 permutations of $i, j, k, l$ yield three independent products of two Kronecker deltas, and we rewrite this equation as

$$
T_{i j k l}=\alpha \delta_{i j} \delta_{k l}+\beta \delta_{i k} \delta_{j l}+\gamma \delta_{i l} \delta_{j k},
$$

where $\alpha, \beta, \gamma$ are linear combinations of the $24 \lambda_{\sigma}$ 's. This should be contrasted with (2.7), which holds for both odd and even $N$.

It should be noted that in general not all products of the form $\Delta_{i_{1} i_{2} \ldots i_{M}} e_{j_{1} j_{2} \ldots j_{N}}(M$ even) are independent. This can be seen immediately by specializing (2.10) to the Cartesian case to give

$$
\sum_{\sigma \in S_{r}}(\operatorname{sgn} \sigma) \Delta_{i_{1} \sigma\left(j_{1}\right) i_{2} \sigma\left(j_{2}\right) \ldots i, \sigma\left(j_{r}\right)}=0 \quad(r \geqslant N+1),
$$

and then contracting with $e_{j_{1} j_{2} \ldots j_{N}}$. For discussion of this in the context of elastic moduli $(N=3)$ see [6].

5. Isotropic tensor fields. Let $\mathcal{M}$ be a differentiable manifold of dimension $N \geqslant 2$ and let $T_{p} \mathcal{M}$ denote the tangent space at a point $p \in \mathcal{M}$. A tensor field of type $(U, V)$ is a section of the tensor bundle

$$
\otimes_{V}^{U} T \mathcal{M}=\underbrace{T \mathcal{M} \otimes \ldots \otimes T M}_{U \text { times }} \otimes T \mathcal{M}^{*} \otimes \ldots \otimes T \mathcal{M}^{*},
$$

i.e. a map $\mathbf{T}: \mathcal{M} \rightarrow \bigotimes_{V}^{U} T \mathcal{M}$ which assigns to each point $p \in \mathcal{M}$ a tensor $\mathbf{T}(p) \epsilon$ $\bigotimes_{V}^{U} T_{p} \mathcal{M}$

Let us suppose that $\mathbf{T}$ is a general isotropic tensor field in the sense that, at each point $p \in \mathcal{M}, \mathbf{T}(p)$ is an isotropic tensor under $G L(N)$. It follows immediately from Section 2 that $U=V$. Further, since the set of general isotropic tensors of type $(U, U)$ forms an invariant vector space over $\mathcal{M}$ generated by the invariant isotropic tensors with components $\Delta_{j_{1} \ldots j_{U}}^{i_{1} \ldots i_{U}}$, we can regard $\mathbf{T}$ as a vector-valued function over $\mathcal{M}$. Hence the components of $\mathbf{T}$ can be written in the form (2.8) with the $\lambda_{\sigma}$ now being scalar fields over $\mathcal{M}$.

We define the derivative of the isotropic tensor field $\mathbf{T}$ of type $(U, U)$ to be the tensor field on $\mathcal{M}$ of type $(U, U+1)$, denoted by $\mathbf{D T}$ and having components $\dagger$

$$
\text { (DT) })_{j_{1} \ldots j_{U} k}^{i_{1} \ldots i_{U}}=\frac{\partial}{\partial x^{k}} T_{j_{1} \ldots j_{U}}^{i_{1} \ldots i_{U}} \equiv \sum_{\sigma \in S_{U}} \frac{\partial \lambda_{\sigma}}{\partial x^{k}} \Delta_{j_{1} \ldots j u}^{\sigma\left(i_{1} \ldots i_{U}\right)}
$$

relative to any local coordinate system $\left(x^{i}\right)$.

By contrast, for any affine connection $\Gamma$ on $\mathcal{M}$, the covariant derivative $\mathbf{D}_{\mathrm{r}} \mathbf{T}$ of $\mathbf{T}$ has

$\dagger$ Note, however, that $\frac{\partial}{\partial x^{k}} T_{j_{1} \ldots j U}^{i_{1} \ldots i}$ are not in general the components of a tensor field if $\mathbf{T}$ is not isotropic. 
components given by

$$
\left(\mathbf{D}_{\Gamma} \mathbf{T}\right)_{j_{1} \ldots j_{U} k}^{i_{1} \ldots i_{U}}=\frac{\partial}{\partial x^{k}} T_{j_{1} \ldots j_{U}}^{i_{1} \ldots i_{U}}+\left.\Gamma_{n k}^{m} T_{j_{1} \ldots j_{U}}^{i_{1} \ldots i_{U}}\right|_{m} ^{n}
$$

where $\left.T_{j_{1} \ldots j_{U}}^{i_{1} \ldots i_{U}}\right|_{p} ^{q}$ denotes the expression on the left-hand side of (3.12) for $W=0$ and $V=U$. Since $\mathbf{T}$ is isotropic the latter term on the right-hand side of (5.2) vanishes, and we obtain

$$
\mathbf{D}_{\Gamma} \mathbf{T}=\mathbf{D T} \text {. }
$$

From (5.3) we deduce that homogeneity of general isotropic tensor fields is independent of any affine structure on $\mathcal{M}$.

Next we show that if $\mathbf{v}$ is any differentiable tangent vector field on $\mathcal{M}$ then the Lie derivative of $\mathbf{T}$ with respect to $\mathbf{v}$ can be written

$$
\mathbf{L}_{\mathbf{v}} \mathbf{T}=(\mathbf{v} . \mathbf{D}) \mathbf{T}
$$

when $\mathbf{T}$ is isotropic, where $\mathbf{v} . \mathbf{D}_{\Gamma}(\cdot)=v^{k}\left[\mathbf{D}_{\Gamma}(\cdot)\right]_{k}$ and similarly for v.D.

To establish (5.4) we note (see [1], for example) that

$$
\left(\mathbf{L}_{\mathbf{v}} \mathbf{T}\right)_{j_{1} \ldots j_{U}}^{i_{1} \ldots i_{U}}=v^{k} \frac{\partial}{\partial x^{k}} T_{j_{1} \ldots j_{U}}^{i_{1} \ldots i_{U}}-\left.\frac{\partial v^{m}}{\partial x^{n}} T_{i_{1} \ldots j_{U}}^{i_{1} \ldots i_{U}}\right|_{m} ^{n}
$$

and, as in the case of (5.2), the latter term in (5.5) vanishes when $\mathbf{T}$ is isotropic.

We now turn to tensor fields that are isotropic under $S L(N)$. Suppose that $\mathcal{M}$ is now an orientable manifold endowed with a volume form e [1] (for example, $\mathcal{M}$ could be an incompressible continuous body); then the set of tensors that are isotropic under $S L(N)$ forms an invariant vector space over $\mathcal{M}$. Let $\phi: \mathcal{M} \rightarrow \mathcal{M}$ be an isochoric (volume preserving) diffeomorphism. Then the tangent map $T \phi$ defines a canonical mapping of isotropic tensors at $p \in \mathcal{M}$ (under $S L(N)$ ) onto the isotropic tensors at $\phi(p)$ (under $S L(N))$. Hence, if $\mathbf{T}$ is now a tensor field of type $(U, V)$ whose value at each point $p$ is an isotropic tensor under $S L(N)$ with weight $W$ we can again regard $\mathbf{T}$ as a vector-valued function over $\mathcal{M}$, with an invariant derivative DT which has components given by (3.9) or (3.10) with $\lambda_{\sigma}$ replaced by $\partial \lambda_{\sigma} / \partial x^{k}$.

If $\Gamma$ is any affine connection on $M$ it is readily shown, using (3.12), that

$$
\mathbf{D}_{\Gamma} \mathbf{T}=\mathbf{D T}+W \mathbf{T} \otimes \gamma,
$$

$\boldsymbol{\gamma}$ being given by $\mathbf{D}_{\Gamma} \mathbf{e}=-\mathbf{e} \otimes \boldsymbol{\gamma}$ when $\mathbf{e}$ is the particular volume form with components (3.7) and satisfying $\mathbf{D e}=\mathbf{0}$. This reduces to (5.3) whenever $\mathbf{D}_{\Gamma} \mathbf{e}=\mathbf{0}$, i.e. when $\Gamma$ is 'volume preserving' (or compatible with the volume form).

Further, for any tangent vector field $\mathbf{v}$ and volume form $\mathbf{e}$,

$$
\mathbf{L}_{\mathbf{v}} \mathbf{T}=\mathbf{v} . \mathbf{D T}-W\left(\operatorname{div}_{\mathbf{e}} \mathbf{v}\right) \mathbf{T},
$$

where, without the need for a connection, $\operatorname{div}_{\mathbf{e}} \mathbf{v}$ is defined by

$$
\mathbf{L}_{\mathbf{v}} \mathbf{e}=\left(\operatorname{div}_{\mathbf{e}} \mathbf{v}\right) \mathbf{e}
$$


The derivatives used above have an important role in continuum mechanics and can be used to simplify certain time derivatives. For example, if $\mathbf{T}$ is a material tensor field isotropic under $G L(3)$ it follows from (5.3) and (5.4) that the material time derivative

$$
\dot{\mathbf{T}}=\frac{\partial \mathbf{T}}{\partial t}+\left(\mathbf{v} . \mathbf{D}_{\Gamma}\right) \mathbf{T}
$$

is identical to the "convected" derivative

$$
\frac{\delta \mathbf{T}}{\delta t}=\frac{\partial \mathbf{T}}{\partial t}+\mathbf{L}_{\mathbf{v}} \mathbf{T},
$$

where $\mathbf{v}$ is the velocity vector field and $\mathbf{D}_{\Gamma}$ is the natural gradient operator in the reference frame. Hence the material time derivative of an isotropic material tensor field is an objective quantity. A similar conclusion holds when $\mathbf{T}$ is isotropic under $S L(3)$, relative to the instantaneous volume form imposed on the body manifold by the reference frame, except that in this case the material time derivative and convected derivative satisfy the (objective) relation

where $\mathbf{D}_{\Gamma} \cdot \mathbf{v}=\operatorname{div}_{\mathbf{e}} \mathbf{v}+\boldsymbol{\gamma} \cdot \mathbf{v}$.

$$
\frac{\delta \mathbf{T}}{\delta t}=\dot{\mathbf{T}}-W\left(\mathbf{D}_{\Gamma} \cdot \mathbf{v}\right) \mathbf{T},
$$

\section{REFERENCES}

1. R. Abraham, J. E. Marsden and T. Ratiu, Manifolds, tensor analysis and applications (Addison Wesley, 1983).

2. S. Golab, Tensor calculus (Elsevier, 1974).

3. H. Jeffreys, On isotropic tensors, Proc. Cambridge Philos. Soc. 73 (1973), 173-176. $339-344$.

4. M. S. Knebelman, Tensors with invariant components, Ann. of Math. (2) 30 (1928),

5. J. G. Oldroyd and B. R. Duffy, Physical constants of a flowing continuum, J. NonNewtonian Fluid Mech. 5 (1979), 141-145.

6. R. W. Ogden, On isotropic tensors and elastic moduli, Proc. Cambridge Philos. Soc. 75 (1974), 427-436.

7. J. A. Schouten, Tensor analysis for physicists, 2nd Edition (Oxford University Press, 1954).

8. T. Y. Thomas, Tensors whose components are absolute constants, Ann. of Math. (2) 27 (1926), 548-550.

UNIVERSITY OF LIVERPOOL

Department of Applied Mathematics and Theoretical Physics

P.O. Box 147

LIVERPOOL L69 3BX

UniVERSITY OF STRATHCLYde

Department of Mathematics

LiVINGSTONE TOWER

26 RICHMOND STREET

GLASGOW G1 1XH
UNIVERSITY OF Glasgow

Department of Mathematics

GlasGow G12 8QW 